VoL. 41 (1990) [249-253]

\title{
SOME INEQUALITIES FOR DIOPHANTINE APPROXIMATION BY CONTINUED FRACTIONS JINGCHENG TONG
}

Let $\xi$ be an irrational number with simple continued fraction expansion $\xi=$ $\left[a_{0} ; a_{1}, a_{2}, \ldots, a_{n}, \ldots\right]$, let $p_{n} / q_{n}$ be its $n$th convergent and let $\theta_{n}=q_{n}\left|q_{n} \xi-p_{n}\right|$. In this paper a general method is introduced to deduce a series of inequalities involving the triple $\left(\theta_{n-1}, \theta_{n}, \theta_{n+1}\right)$.

\section{INTRODUCTION}

Let $\xi$ be an irrational number with simple continued fraction expansion $\xi=$ $\left[a_{0} ; a_{1}, a_{2}, \ldots, a_{n}, \ldots\right]$, where $a_{0}$ is an integer and the $a_{i}(i=1,2, \ldots)$ are positive integers. Let $p_{n} / q_{n}$ denote the $n$th convergent. The sequence of approximation constants is defined as follows.

$$
\theta_{n}=q_{n}\left|q_{n} \xi-p_{n}\right|
$$

About forty years ago, Brauer and Macon $[1,4]$ proved the following inequalities involving the triple $\left(\theta_{n-1}, \theta_{n}, \theta_{n+1}\right)$ :

$$
\begin{aligned}
\theta_{n-1} \theta_{n} \theta_{n+1} & <4 / 27, \\
\theta_{n-1}+\theta_{n}+\theta_{n+1} & <2, \\
\frac{1}{\theta_{n-1}}+\frac{1}{\theta_{n}}+\frac{1}{\theta_{n+1}} & >6 .
\end{aligned}
$$

Recently, Jager and Kraaikamp [2] generalised the above results. They proved the following inequalities which include (2), (3) and (4) for $a_{n+1}=1$.

$$
\begin{aligned}
& \theta_{n-1} \theta_{n} \theta_{n+1}<\left(a_{n+1}+1\right)^{2} /\left(a_{n+1}+2\right)^{3}, \\
& \theta_{n-1}+\theta_{n}+\theta_{n+1}<\left(2 a_{n+1}+3\right) /\left(a_{n+1}+2\right), \\
& \frac{1}{\theta_{n-1}}+\frac{1}{\theta_{n}}+\frac{1}{\theta_{n+1}}>4+a_{n+1}+\frac{2}{a_{n+1}+1} .
\end{aligned}
$$

In this paper, using the idea in $[7,8]$, we introduce a general method for deducing a series of inequalities involving the triple $\left(\theta_{n-1}, \theta_{n}, \theta_{n+1}\right)$. The results cited above are very special cases of the new inequalities we obtain.

Received 17 April, 1989

The author sincerely thanks Professor H. Jager and Professor C. Kraaikamp for their very valuable correspondence.

Copyright Clearance Centre, Inc. Serial-fee code: 0004-9729/90 \$A2.00+0.00. 


\section{Preliminaries}

Let $\xi=\left[a_{0} ; a_{1}, a_{2}, \ldots, a_{n}, \ldots\right], \alpha_{n+1}=\left[a_{n+1} ; a_{n+2}, \ldots\right]$. Then $($ see $[5,6])$ we have

$$
\xi-\frac{p_{n}}{q_{n}}=\frac{\alpha_{n+1} p_{n}+p_{n-1}}{\alpha_{n+1} q_{n}+q_{n-1}}-\frac{p_{n}}{q_{n}}=\frac{(-1)^{n}}{\left(\alpha_{n+1}+q_{n-1} / q_{n}\right) q_{n}^{2}} .
$$

Since $q_{n-1} / q_{n}=\left[0, a_{n}, a_{n-1}, \ldots, a_{1}\right]$, we have that

$$
\theta_{n}=q_{n}\left|q_{n} \xi-p_{n}\right|=1 /\left(\left[a_{n+1} ; a_{n+2}, \ldots\right]+\left[0 ; a_{n}, a_{n-1}, \ldots, a_{1}\right]\right) .
$$

Set $P=\left[a_{n+2} ; a_{n+3}, \ldots\right], Q=\left[a_{n} ; a_{n-1}, \ldots, a_{1}\right]$ and $\Delta=a_{n+1} P Q+P+Q$. Then we have the following relations.

$$
\begin{gathered}
\theta_{n-1}=\left(Q+\frac{1}{a_{n+1}+P^{-1}}\right)^{-1}=\frac{a_{n+1} P+1}{a_{n+1} P Q+P+Q}=\frac{a_{n+1} P+1}{\Delta}, \\
\theta_{n}=\left(a_{n+1}+P^{-1}+Q^{-1}\right)^{-1}=\frac{P Q}{a_{n+1} P Q+P+Q}=\frac{P Q}{\Delta} \\
\theta_{n+1}=\left(P+\frac{1}{a_{n+1}+Q^{-1}}\right)^{-1}=\frac{a_{n+1} Q+1}{a_{n+1} P Q+P+Q}=\frac{a_{n+1} Q+1}{\Delta} .
\end{gathered}
$$

Viewing $P$ and $Q$ as variables, one easily finds the following formal partial derivatives with respect to $P, Q$.

$$
\begin{aligned}
\theta_{n-1, P}^{\prime} & =-1 / \Delta^{2} ; \quad \theta_{n-1, Q}^{\prime}=-\left(a_{n+1} P+1\right)^{2} / \Delta^{2}, \\
\theta_{n, P}^{\prime} & =Q^{2} / \Delta^{2} ; \quad \theta_{n, Q}^{\prime}=P^{2} / \Delta^{2}, \\
\theta_{n+1, P}^{\prime} & =-\left(a_{n+1} Q+1\right)^{2} / \Delta^{2} ; \quad \theta_{n+1, Q}^{\prime}=-1 / \Delta^{2} .
\end{aligned}
$$

\section{Main Results}

We need a very simple lemma (one need only observe that $P>a_{n+2}$ and $Q>a_{n}$ ).

Lemma 1. Let $f\left(\theta_{n-1}, \theta_{n}, \theta_{n+1}\right)$ be a function defined for $\theta_{n-1}, \theta_{n}, \theta_{n+1}>0$. Let $\Delta_{0}=a_{n} a_{n+1} a_{n+2}+a_{n}+a_{n+2}$. Then

(i) if $f\left(\left(a_{n+1} P+1\right) / \Delta,(P Q) / \Delta,\left(a_{n+1} Q+1\right) / \Delta\right)$ is a decreasing function in both $P$ and $Q$, we have

$$
f\left(\theta_{n-1}, \theta_{n}, \theta_{n+1}\right)<f\left(\frac{a_{n+1} a_{n+2}+1}{\Delta_{0}}, \frac{a_{n} a_{n+2}}{\Delta_{0}}, \frac{a_{n+1} a_{n+2}+1}{\Delta_{0}}\right)
$$

(ii) if $f\left(\left(a_{n+1} P+1\right) \Delta,(P Q) / \Delta,\left(a_{n+1} Q+1\right) / \Delta\right)$ is an increasing function in both $P$ and $Q$, we have

$$
f\left(\theta_{n-1}, \theta_{n}, \theta_{n+1}\right)>f\left(\frac{a_{n+1} a_{n+2}+1}{\Delta_{0}}, \frac{a_{n} a_{n+2}}{\Delta_{0}}, \frac{a_{n+1} a_{n+2}+1}{\Delta_{0}}\right) .
$$


Since $a_{n} \geqslant 1, a_{n+2} \geqslant 1,(14)$ and (15) can be replaced by

$$
\begin{aligned}
& f\left(\theta_{n-1}, \theta_{n}, \theta_{n+1}\right)<f\left(\frac{a_{n+1}+1}{a_{n+1}+2}, \frac{1}{a_{n+1}+2}, \frac{a_{n+1}+1}{a_{n+1}+2}\right), \\
& f\left(\theta_{n-1}, \theta_{n}, \theta_{n+1}\right)>f\left(\frac{a_{n+1}+1}{a_{n+1}+2}, \frac{1}{a_{n+1}+2}, \frac{a_{n+1}+1}{a_{n+1}+2}\right) .
\end{aligned}
$$

THEOREM 1. If $\alpha, \gamma \geqslant \beta>0$, then

$$
\theta_{n-1}^{\alpha} \theta_{n}^{\beta} \theta_{n+1}^{\gamma}<\frac{\left(a_{n+1}+1\right)^{\alpha+\gamma}}{\left(a_{n+1}+2\right)^{\alpha+\beta+\gamma}}
$$

PROOF: Let $f\left(\theta_{n-1}, \theta_{n}, \theta_{n+1}\right)=\theta_{n-1}^{\alpha} \theta_{n}^{\beta} \theta_{n+1}^{\gamma}$. Then

$$
\begin{aligned}
(\log f)_{P}^{\prime} & =\frac{\alpha}{\theta_{n-1}} \theta_{n-1, P}^{\prime}+\frac{\beta}{\theta_{n}} \theta_{n, P}^{\prime}+\frac{\gamma}{\theta_{n+1}} \theta_{n+1, P}^{\prime} \\
& =\frac{-\alpha}{\left(a_{n+1} P+1\right) \Delta}+\frac{\beta Q}{P \Delta}+\frac{-\gamma\left(a_{n+1} Q+1\right)}{\Delta} \\
& <\frac{\beta Q}{\Delta}-\frac{\gamma Q}{\Delta}<0 .
\end{aligned}
$$

Similarly one can show that $(\log f)_{Q}^{\prime}<0$. Therefore $f\left(\theta_{n-1}, \theta_{n}, \theta_{n+1}\right)$ is a decreasing function in $P$ and $Q$. By Lemma 1 and (14') we have (16).

Letting $\alpha=\beta=\gamma=1$, we have inequality (5).

ThEOREM 2. If $3 \geqslant \alpha, \gamma \geqslant \beta \geqslant 1, A, B, C>0$, and $\alpha A, \gamma C \geqslant \beta B$, then

(17) $A \theta_{n-1}^{\alpha}+B \theta_{n}^{\beta}+C \theta_{n+1}^{\gamma}<A\left(\frac{a_{n+1}+1}{a_{n+1}+2}\right)^{\alpha}+B\left(\frac{1}{a_{n+1}+2}\right)^{\beta}+C\left(\frac{a_{n+1}+1}{a_{n+1}+2}\right)^{\gamma}$.

PROOF: Let $f\left(\theta_{n-1}, \theta_{n}, \theta_{n+1}\right)=A \theta_{n-1} \alpha+B \theta_{n}^{\beta}+C \theta_{n+1}^{\gamma}$. Then

$$
\begin{aligned}
f_{P}^{\prime} & =\alpha A \theta_{n-1}^{\alpha-1} \theta_{n-1, P}^{\prime}+\beta B \theta_{n}^{\beta-1} \theta_{n, P}^{\prime}+\gamma C \theta_{n+1}^{\gamma-1} \theta_{n+1, P}^{\prime} \\
& =\frac{-\alpha A}{\left(a_{n+1} P+1\right)^{\alpha-1} \Delta^{3-\alpha}}+\frac{B Q^{3-\beta}}{P^{\beta-1} \Delta^{3-\beta}}+\frac{-\gamma C\left(a_{n+1} Q+1\right)^{3-\gamma}}{\Delta^{3-\gamma}} \\
& <\frac{\beta B Q^{3-\beta}}{\Delta^{3-\beta}}-\frac{\gamma C Q^{3-\gamma}}{\Delta^{3-\gamma}} \\
& =\Delta^{\beta-3}\left(\beta B Q^{3-\beta}-\Delta^{\gamma-\beta} \gamma C Q^{3-\gamma}\right) .
\end{aligned}
$$

Since $\gamma \geqslant \beta$, we have $\Delta^{\gamma-\beta}=\left(a_{n+1} P Q+P+Q\right)^{\gamma-\beta}>Q^{\gamma-\beta}$, hence

$$
f_{P}^{\prime}<\frac{Q^{3-\beta}}{\Delta^{3-\beta}}(\beta B-\gamma C)<0 .
$$


Similarly, one can show that $f_{Q}^{\prime}<0$. By Lemma 1 and $\left(14^{\prime}\right)$, we have inequality (17).

Letting $A=B=C=1$ and $\alpha=\beta=\gamma=1$, we have inequality (6).

Theorem 3. Let $\alpha, \gamma \geqslant 1 \geqslant \beta>0$, let $A, B, C>0$ and $\alpha A, \gamma C \geqslant \beta B$. Then

$$
\frac{A}{\theta_{n-1}^{\alpha}}+\frac{B}{\theta_{n}^{\beta}}+\frac{C}{\theta_{n+2}^{\gamma}}>A\left(\frac{a_{n+1}+2}{a_{n+1}+1}\right)^{\alpha}+B\left(a_{n+2}+2\right)^{\beta}+C\left(\frac{a_{n+2}+2}{a_{n+1}+1}\right)^{\gamma}
$$

Proof: Let $f\left(\theta_{n-1}, \theta_{n}, \theta_{n+1}\right)=A \theta_{n-1}^{-\alpha}+B \theta_{n}^{-\beta}+C \theta_{n+1}^{-\gamma}$. Then

$$
\begin{aligned}
f_{P}^{\prime} & =\frac{-\alpha A}{\theta_{n-1}^{\alpha+1}} \theta_{n-1, P}^{\prime}+\frac{-\beta B}{\theta_{n}^{\beta+1}} \theta_{n, P}^{\prime}+\frac{-\gamma C}{\theta_{n+1}^{\gamma+1}} \theta_{n+1, P}^{\prime} \\
& =\frac{\alpha A \Delta^{\alpha-1}}{\left(a_{n+1} P+1\right)^{\alpha+1}}-\frac{\beta B \Delta^{\beta-1}}{P^{\beta+1} Q^{\beta-1}}+\frac{\gamma C \Delta^{\gamma-1}}{\left(a_{n+1} Q+1\right)^{\gamma-1}} \\
& >\frac{\gamma C \Delta^{\gamma-1}}{\left(a_{n+1} Q+1\right)^{\gamma-1}}-\frac{\beta B Q^{1-\beta}}{P^{\beta+1} \Delta^{1-\beta}}
\end{aligned}
$$

Since $\Delta=a_{n-1} P Q+P+Q>a_{n+1} Q+1>Q$, we have $f_{P}^{\prime}>\gamma C-\beta B \geqslant 0$.

Similarly one can show that $f_{Q}^{\prime}>0$. By Lemma 1 and $\left(15^{\prime}\right)$, we have inequality (18).

Letting $\alpha=\beta=\gamma=1$ and $A=B=C=1$ in Theorem 3, we have inequality (7).

TheOREM 4 . Let $A, C \geqslant B \geqslant 1$. Then

$$
A^{\theta_{n-1}} B^{\theta_{n}} C^{\theta_{n+1}}<A^{\frac{a_{n+1}+1}{a_{n+1}+2}} B^{\frac{1}{a_{n+1}+2}} C^{\frac{a_{n+1}+1}{a_{n+1}+2}}
$$

PRoOF: Let $f\left(\theta_{n-1}, \theta_{n}, \theta_{n+1}\right)=A^{\theta_{n-1}} B^{\theta_{n}} C^{\theta_{n+1}}$. Then

$$
\begin{aligned}
(\log f)_{P}^{\prime} & =\theta_{n-1, P}^{\prime} \log A+\theta_{n, P}^{\prime} \log B+\theta_{N+1, P}^{\prime} \log C \\
& =\frac{1}{\Delta^{2}}\left(-\log A+Q^{2} \log B-\left(a_{n+1} Q+1\right)^{2} \log C\right) \\
& <\frac{1}{\Delta^{2}}\left(Q^{2} \log B-Q^{2} \log C\right) \\
& <0
\end{aligned}
$$

Similarly one can show that $f_{Q}^{\prime}<0$. By Lemma 1 and $\left(14^{\prime}\right)$, we have inequality (19).

Letting $A=B=C$, we again have inequality (6). 
The significance of Theorems $1,2,3$, and 4 is not just that of their statements. They are examples of a general method. For a given function $f\left(\theta_{n-1}, \theta_{n}, \theta_{n+1}\right)$ involving parameters, one first finds $f_{P}^{\prime}$ and $f_{Q}^{\prime}$ by using expressions (8)-(13) (this is the decisive step), then one considers proper conditions on the parameters to make $f$ decreasing or increasing in $P$ or $Q$. In this way one may deduce numerous other inequalities involving the triple $\left(\theta_{n-1}, \theta_{n}, \theta_{n+1}\right)$.

\section{REFERENCES}

[1] A. Brauer and N. Macon, 'On the approximation of irrational numbers by the convergents of their continued fractions', Amer. J. Math. 71 (1949), 349-361. 72 (1950), 419-424 .

[2] H. Jager and C. Kraaikamp, 'On the approximation by continued fractions', Indag. Math. (to appear).

[3] C. Kraaikamp, 'On the approximation by continued fractions II', preprint.

[4] N. Macon, 'Some theorems on the approximation of irrational numbers by the convergents of their continued fractions', J. Elisha Mitchell Sci. Soc. 67 (1951), 99-107.

[5] O. Perron, Die Lehre von den Kettenbrüchen I, II, 3rd. ed. (Teubner, 1954).

[6] W. Schmidt, Diophantine Approximations, Lecture Notes in Math. 785 (Springer-Verlag, Berlin, Heidelberg, New York, 1980).

[7] J. Tong, 'The conjugate property of the Borel theorem on Diophantine approximation', Math. Z. 184 (1983), 151-153.

[8] J. Tong, 'The conjugate property for Diophantine approximation of continued fractions', Proc. Amer. Math. Soc. 105 (1989). 
